\title{
THE PYTHAGOREAN THEOREM IN CERTAIN SYMMETRY CLASSES OF TENSORS
}

\author{
BY \\ MARVIN MARCUS( $\left.{ }^{1}\right)$ AND HENRYK MINC( $\left.{ }^{2}\right)$
}

The Hadamard determinant theorem [1] states that if $A=\left(a_{i j}\right)$ is a positive semi-definite $k$-square Hermitian matrix then

$$
\operatorname{det} A \leqq \prod_{i=1}^{k} a_{i i}
$$

with equality if and only if $A$ has a zero row or $A$ is diagonal.

In a recent paper [3] it was conjectured that an analogous result to (1) holds for the permanent of $A$. We recall that the permanent is defined by

$$
\operatorname{per}(A)=\sum_{\sigma \in S_{k}} \prod_{i=1}^{k} a_{\sigma(i) i}
$$

where the summation is over the whole symmetric group of degree $k$. Recent interest in the permanent function stems from its application to a variety of combinatorial problems [4] and a partly unresolved conjecture of B. L. van der Waerden [2]. In [3] it was suggested that if $A$ is once again a positive semi-definite $k$-square Hermitian matrix then

$$
\operatorname{per}(A) \geqq \prod_{i=1}^{k} a_{i j}
$$

with equality if and only if $A$ has a zero row or $A$ is diagonal. We are as yet unable to prove this but the subsequent inequality (3) is a step in this direction.

The first purpose of this note is to exhibit (1) as a case of the Pythagorean Theorem in a suitable symmetry class of tensors. Of course, many proofs of (1) are extant and our purpose in reproving it here is to exhibit a technique that is proving itself useful for examining a wide variety of matrix functions. We then show by a similar approach that

$$
\operatorname{per}(A) \geqq\left(\prod_{i=1}^{k} a_{i i}\right) k ! / k^{2 k}
$$

where the inequality is strict unless $A$ has a zero row.

Presented to the Society, November 18, 1961; received by the editors October 23, 1961.

(1) The work of this author was supported in part by the Office of Naval Research.

(2) The work of this author was supported by the Research Council, University of Florida. 
The technique here is to regard the determinant and permanent functions as analytic expressions for inner products in suitable symmetry classes of tensors. To be explicit, let $U$ be an $n$-dimensional unitary vector space with an inner product $(x, y)$. Let $U^{(k)}$ be the space of $k$-tensors over $U$; i.e., the $n^{k}$-dimensional dual space of the vector space $M^{(k)}$ of multilinear functionals $\phi$ of $k$-tuples of vectors from $U$. Certain distinguished "pure" vectors in $U^{(k)}$ are denoted by $f=x_{1} \otimes \cdots \otimes x_{k}$ where $x_{i} \in U$ and $f$ is defined by $f(\phi)$ $=\phi\left(x_{1}, \cdots, x_{k}\right)$ for each $\phi \in M^{(k)}$. The pure vectors span $U^{(k)}$ and the conjugate bilinear functional defined on pure vectors by

$$
\left(x_{1} \otimes \cdots \otimes x_{k}, y_{1} \otimes \cdots \otimes y_{k}\right)=\prod_{i=1}^{k}\left(x_{i}, y_{i}\right)
$$

is extendable to a unitary inner product on $U^{(k)}$. Let $T$ and $S$ be the symmetry operators of $U^{(k)}$ into itself defined by

$$
\begin{aligned}
& T\left(x_{1} \otimes \cdots \otimes x_{k}\right)=\frac{1}{k !} \sum_{\sigma \in S_{k}} \epsilon(\sigma) x_{\sigma(1)} \otimes \cdots \otimes x_{\sigma(k)}, \\
& S\left(x_{1} \otimes \cdots \otimes x_{k}\right)=\frac{1}{k !} \sum_{\sigma \in S_{k}} x_{\sigma(1)} \otimes \cdots \otimes x_{\sigma(k)},
\end{aligned}
$$

where $\epsilon(\sigma)= \pm 1$ according as $\sigma$ is even or odd.

It is well known that $T$ and $S$ are Hermitian (with respect to the inner product in (4)) and idempotent. Moreover, one computes easily that

$$
\left(T x_{1} \otimes \cdots \otimes x_{k}, y_{1} \otimes \cdots \otimes y_{k}\right)=\frac{1}{k !} \operatorname{det}\left(\left(x_{i}, y_{j}\right)\right)
$$

and

$$
\left(S x_{1} \otimes \cdots \otimes x_{k}, y_{1} \otimes \cdots \otimes y_{k}\right)=\frac{1}{k !} \operatorname{per}\left(\left(x_{i}, y_{j}\right)\right)
$$

To prove (1) we first remark that if $A$ is singular, $\operatorname{det} A=0$, then the inequality obviously holds and equality requires that some $a_{i i}=0$. But then $0 \leqq a_{i i} a_{j j}-\left|a_{i j}\right|^{2}=-\left|a_{i j}\right|^{2}$ and hence $a_{i j}=0, j=1, \cdots, k$, and row $i$ of $A$ is zero. Hence assume $A$ is nonsingular and let $x_{1}, \cdots, x_{k}$ be a set of linearly independent vectors such that $\left(x_{i}, x_{j}\right)=a_{i j}, i, j=1, \cdots, k$. Let $u_{1}, \cdots, u_{k}$ be the E. Schmidt orthonormalizing sequence for $x_{1}, \cdots, x_{k}$. That is, the space $\left\langle x_{1}, \cdots, x_{p}\right\rangle$ spanned by $x_{1}, \cdots, x_{p}$ is the same as the space $\left\langle u_{1}, \cdots, u_{p}\right\rangle$ spanned by $u_{1}, \cdots, u_{p}, p=1, \cdots, k$. Then since $u_{i_{1}} \otimes \cdots \otimes u_{i_{k}}, 1 \leqq i_{\alpha} \leqq k$, $\alpha=1, \cdots, k$, is an orthonormal basis in $U^{(k)}$ we have from the Pythagorean theorem that 


$$
\begin{aligned}
\frac{1}{k !} \operatorname{det} A & =\frac{1}{k !} \operatorname{det}\left(\left(x_{i}, x_{j}\right)\right)=\left(T x_{1} \otimes \cdots \otimes x_{k}, T x_{1} \otimes \cdots \otimes x_{k}\right) \\
& =\sum\left|\left(T x_{1} \otimes \cdots \otimes x_{k}, u_{i_{1}} \otimes \cdots \otimes u_{i_{k}}\right)\right|^{2} \\
& =\left(\frac{1}{k !}\right)^{2} \sum\left|\operatorname{det}\left(\left(x_{s}, u_{i_{i}}\right)\right)\right|^{2}, \quad s, t=1, \cdots, k,
\end{aligned}
$$

where the summation extends over all $k^{k}$ ordered selections $\left(i_{1}, \cdots, i_{k}\right)$ from $1, \cdots, k$. Since the determinant vanishes when two columns are the same, the last summation may be taken over sets of distinct ordered choices $\left(i_{1}, \cdots, i_{k}\right)$, i.e., over all $k$ ! permutations of $1, \cdots, k$. Hence

$$
\begin{aligned}
& \frac{1}{k !} \operatorname{det} A=\left(\frac{1}{k !}\right)^{2} \sum_{\sigma \in S_{k}}\left|\operatorname{det}\left(\left(x_{s}, u_{\sigma(t)}\right)\right)\right|^{2} \\
& =\frac{1}{k !}\left|\operatorname{det}\left(\left(x_{e}, u_{t}\right)\right)\right|^{2} \\
& =\frac{1}{k !}\left|\operatorname{det}\left[\begin{array}{cccc}
\left(x_{1}, u_{1}\right) & 0 & \cdots & 0 \\
\left(x_{2}, u_{1}\right) & \left(x_{2}, u_{2}\right) & 0 \cdots & 0 \\
\vdots & & & \vdots \\
\left(x_{k}, u_{1}\right) & \left(x_{k}, u_{2}\right) & \cdots & \left(x_{k}, u_{k}\right)
\end{array}\right]\right|^{2} \\
& =\frac{1}{k !} \prod_{i=1}^{k}\left|\left(x_{i}, u_{i}\right)\right|^{2} \\
& \leqq \frac{1}{k !} \prod_{i=1}^{k}\left(x_{i}, x_{i}\right)=\frac{1}{k !} \prod_{i=1}^{k} a_{i i}
\end{aligned}
$$

Now the equality holds by Schwarz's inequality if and only if $x_{\alpha}$ is a multiple of $u_{\alpha}$. But since $u_{1}, \cdots, u_{k}$ is an orthonormal set, it follows that $A=\left(\left(x_{i}, x_{j}\right)\right)$ is diagonal.

From (6) we compute that

$$
\text { per } \begin{aligned}
A & =k !\left(S x_{1} \otimes \cdots \otimes x_{k}, x_{1} \otimes \cdots \otimes x_{k}\right) \\
& =k !\left\|S x_{1} \otimes \cdots \otimes x_{k}\right\|^{2} \\
& \geqq k !\left|\left(S x_{1} \otimes \cdots \otimes x_{k}, u \otimes \cdots \otimes u\right)\right|^{2} \\
& =k !\left|\left(x_{1} \otimes \cdots \otimes x_{k}, S u \otimes \cdots \otimes u\right)\right|^{2} \\
& =k !\left|\left(x_{1} \otimes \cdots \otimes x_{k}, u \otimes \cdots \otimes u\right)\right|^{2} \\
& =k ! \prod_{i=1}^{k}\left|\left(x_{i}, u\right)\right|^{2}
\end{aligned}
$$


where $u$ is any unit vector in $U$.

The problem then is to find for fixed $x_{i}, i=1, \cdots, k$, a significant lower bound for the expression $\prod_{i=1}^{k}\left|\left(x_{i}, u\right)\right|^{2}$ as a function of the unit vector $u$. We have the

Lemma. If $x_{1}, \cdots, x_{k}$ are vectors then there exists a unit vector $u$ such that

$$
\left|\left(x_{s}, u\right)\right| \geqq\left\|x_{s}\right\| / k, \quad s=1, \cdots, k .
$$

Proof. Let $y_{i}=x_{i} /\left\|x_{i}\right\|$ or $-x_{i} /\left\|x_{i}\right\|$ so that $\sum_{i=1}^{k} y_{i}$ is of maximal length. That is, $\left\|\sum_{i=1}^{k} y_{i}\right\| \geqq\left\|\sum_{i=1}^{k} \pm x_{i} /\right\| x_{i}\|\|$ for all choices of signs on the $x_{i}$. We assert that

$$
\operatorname{Re}\left(y_{s}, \sum_{i=1, i \neq s}^{k} y_{i}\right) \geqq 0, \quad s=1, \cdots, k .
$$

For let $z_{\imath}=\sum_{i=1, i \neq s}^{k} y_{i}$ and $z=\sum_{i=1}^{k} y_{i}$ and we have

$$
\begin{gathered}
\|z\|^{2}=\left\|y_{s}+z_{s}\right\|^{2} \geqq\left\|-y_{s}+z_{s}\right\|^{2}, \\
\left\|y_{s}\right\|^{2}+2 \operatorname{Re}\left(y_{s}, z_{s}\right)+\left\|z_{s}\right\|^{2} \geqq\left\|y_{s}\right\|^{2}-2 \operatorname{Re}\left(y_{s}, z_{s}\right)+\left\|z_{s}\right\|^{2}
\end{gathered}
$$

and (8) follows.

It is clear that

$$
\|z\| \leqq \sum_{i=1}^{k}\left\|y_{i}\right\|=k
$$

and thus

$$
\begin{aligned}
\left|\left(y_{s}, \frac{z}{\|z\|}\right)\right| & =\left|\left(y_{s}, \frac{y_{s}+z_{s}}{\|z\|}\right)\right| \\
& =\frac{1}{\|z\|}\left|\left(\left\|y_{8}\right\|^{2}+\left(y_{s}, z_{s}\right)\right)\right| \\
& \geqq \frac{1}{\|z\|} \operatorname{Re}\left(1+\left(y_{s}, z_{s}\right)\right) \geqq \frac{1}{\|z\|} \geqq \frac{1}{k} .
\end{aligned}
$$

In (7) we take $u=z /\|z\|$ to obtain

$$
\text { per } \begin{aligned}
A & \geqq k ! \prod_{i=1}^{k}\left|\left(y_{i}, u\right)\right|^{2}\left\|x_{i}\right\|^{2} \\
& \geqq\left(k ! / k^{2 k}\right) \prod_{i=1}^{k}\left\|x_{i}\right\|^{2} \\
& =\left(k ! / k^{2 k}\right) \prod_{i=1}^{k} a_{i i}
\end{aligned}
$$


Clearly if any $x_{j}=0$ then per $A=0, a_{j j}=0$ and (3) is equality. If no $x_{j}=0$ then (9) can be equality only if $z=\theta k y_{s}$, where $|\theta|=1, s=1, \cdots, k$, in which case

$$
\left|\left(y_{s}, u\right)\right|=\left|\left(y_{s}, \frac{z}{\|z\|}\right)\right|=k / k=1>\frac{1}{k}
$$

and hence the inequality in the lemma is strict for $k>1$.

Actually a refinement of the above argument shows that

$$
\prod_{s=1}^{k}\left|\left(y_{s}, u\right)\right| \geqq \frac{k^{1 / 2}}{k} \frac{k^{1 / 2}-1}{k-1} \cdots \frac{k^{1 / 2}-r}{k-r} \frac{1}{k^{k-r-1}},
$$

where $r=\left[k /\left(k^{1 / 2}+1\right)\right]$, and therefore,

$$
\operatorname{per}(A) \geqq \prod_{i=1}^{k} a_{i i} k ! /\left(\frac{k^{1 / 2}}{k} \frac{k^{1 / 2}-1}{k-1} \cdots \frac{k^{1 / 2}-r}{k-r} \frac{1}{k^{k-r-1}}\right)^{2} .
$$

For

$$
\|z\|^{2}=(z, z)=\sum_{s=1}^{k}\left(y_{s}, z\right)=k+\sum_{s=1}^{k}\left(y_{s}, z_{s}\right)=k+\sum_{s=1}^{k} \operatorname{Re}\left(y_{s}, z_{s}\right) \geqq k,
$$

i.e., $\|z\| \geqq k^{1 / 2}$. We can assume without loss of generality that $\operatorname{Re}\left(y_{1}, z\right) \geqq \cdots$ $\geqq \operatorname{Re}\left(y_{k}, z\right)$. Now from (11) we have

$$
\|z\|=\sum_{s=1}^{k} \operatorname{Re}\left(y_{\bullet}, \frac{z}{\|z\|}\right) \geqq k^{1 / 2}
$$

and therefore $\operatorname{Re}\left(y_{1}, z /\|z\|\right) \geqq k^{1 / 2} / k$, and a fortiori, $\left|\left(y_{1}, z /\|z\|\right)\right| \geqq k^{1 / 2} / k$. Further

$$
\left|\sum_{s=2}^{k}\left(y_{s}, \frac{z}{\|z\|}\right)\right| \geqq\left|\sum_{s=1}^{k}\left(y_{s}, \frac{z}{\|z\|}\right)\right|-\left|\left(y_{1}, \frac{z}{\|z\|}\right)\right| \geqq k^{1 / 2}-1
$$

and thus $\left|\left(y_{2}, z /\|z\|\right)\right| \geqq\left(k^{1 / 2}-1\right) /(k-1)$.

In general

$$
\left|\left(y_{s+1}, \frac{z}{\|z\|}\right)\right| \geqq \frac{k^{1 / 2}-s}{k-s} .
$$

Comparing (12) with (10) we find, by an elementary computation, that

$$
\max \left\{\frac{k^{1 / 2}-s}{k-s}, \frac{1}{k}\right\}=\left\{\begin{array}{cl}
\frac{k^{1 / 2}-s}{k-s} & \text { for } s \leqq r \\
\frac{1}{k} & \text { for } s>r
\end{array}\right.
$$

where $r$ is the greatest integer in $k /\left(k^{1 / 2}+1\right)$. 
Thus (12) gives a better lower bound for $s=1, \cdots, r$ while (10) gives a better lower bound for $s=r+1, \cdots, k$. The result follows.

\section{REFERENCES}

1. J. Hadamard, Resolution d'une question relative aux déterminants, Bull. Sci. Math. (2) 17 (1893), 240-248.

2. Marvin Marcus and Morris Newman, The permanent function as an inner product, Bull. Amer. Math. Soc. 67 (1961), 223-224.

3. - Inequalities for the permanent function, Ann. of Math. 75 (1962), 47-62.

4. H. J. Ryser, Compound and induced matrices in combinatorial analysis, Proc. Sympos. Appl. Math. Vol. 10, pp. 149-168, Amer. Math. Soc., Providence, R. I., 1960.

National BurEaU OF STANDARDS,

WASHINGTON, D. C.

The University of British Columbia,

VANCOUver, British Columbia

UNIVERSITY OF FLORIDA,

Gainesville, FLORIDA 